Check for updates

1 The Health Foundation

2 The BMJ

@drcatchatfield

Cite this as: BMJ2022;376:0475

http://dx.doi.org/10.1136/bmj.0475

Published: 24 February 2022

\section{Lessons in quality improvement}

\section{The Health Foundation and The BMJ introduce a collection of articles to support quality improvement measures}

\section{Bryan Jones, ${ }^{1}$ Cat Chatfield ${ }^{2}$}

For 30 years, quality improvement approaches and tools have been used to improve the way in which healthcare is organised and delivered. In the UK, use of systematic improvement approaches, such as PDSA (plan, do, study, act) cycles or Lean, ${ }^{12}$ is now relatively common, and many clinicians are introduced to quality improvement concepts at an early stage in their training.

Many quality improvement interventions have had a positive impact on patients-for example by standardising care processes and pathways and removing delays and duplication. ${ }^{3}$ They have given clinicians and other professionals the opportunity to address the problems in care quality that matter most to them and their patients. However, not every quality improvement intervention is successful.

Some fail to achieve their desired outcomes or are not sustained. 4 This is not surprising given the experimental nature of quality improvement, and often the learning from this experience better prepares participants for their next intervention. Other interventions fall short because those involved are taking part under obligation and are unconvinced of the interventions' purpose and value. Meanwhile, some interventions may implement a solution that runs counter to standard practice elsewhere, causing a safety risk or creating unintended consequences in another part of an organisation or system, and so actually have a negative impact on patient care.

To help teams and organisations maximise their chance of planning and delivering successful and sustained quality improvement interventions, the $B M J$, in partnership with and funded by the Health Foundation, has created a collection of essays, analysis, and education articles on key quality improvement topics. The collection highlights the skills and knowledge needed to do quality improvement well, and the relational, cultural, infrastructural, and strategic challenges that have to be addressed for improvement to flourish in healthcare settings and beyond.

One of the most important insights to emerge from the collection is that no improvement intervention should be conducted in isolation. ${ }^{5} \mathrm{~A}$ weakness of much quality improvement work to date is that it tends to be carried out as a "highly local, almost artisan activity" focused on discrete "small, time limited projects." 6 Such projects often absorb considerable amounts of time, effort, and energy, but may make only a marginal, and sometimes fleeting, difference to patient care. To avoid this, any intervention needs to be integrated and aligned with the overarching strategic approach to improvement of the organisation or system in which it is set. ${ }^{7}$
Moreover, the job of improving care should not rest solely with front line clinical teams: it requires active and consistent leadership from within the organisation and system, ${ }^{8}$ engaged and supportive managers, and the involvement from the start of patients and citizens as co-producers of improvement. ${ }^{910}$ This is happening in some places but is still far from being the norm.

Another challenge for healthcare systems highlighted in the collection is how to marshal and deploy the collective improvement expertise and knowledge of those doing improvement to identify and address quality issues that require a pathway or system-wide response. One solution is to create and resource networks and platforms that bridge organisational and sectoral boundaries and give people the space to come together and share ideas and expertise, learn from each other, and tackle quality challenges collaboratively. ${ }^{11-13}$ With interest in employing quality improvement approaches at population health level now on the rise, ${ }^{14}$ the need for such large scale engagement platforms, together with people who can act as "system integrators," 1516 is becoming ever more apparent. With patients and carers often the only people who experience care across the system, it is critical to integrate them as a "vital source of different perspectives" 10 in healthcare improvement.

Working at organisation or system level is not easy. It is precisely for this reason that so much improvement to date has taken place within specific teams and services. Organisations such as hospitals are complex entities with "multiple" and

"overlapping subgroups." ${ }^{17}$ And while some "cultural attributes are widespread and stable," others may be shared only in sub groups or held only tentatively."17 Equally, many processes and pathways are not stable, but are in a constant state of flux. ${ }^{11}$

For anyone involved in improvement, an understanding of the social and cultural complexity of healthcare organisations, coupled with a recognition of how change happens in complex systems, is likely to be an increasingly valuable skill. After all, no other public or commercial sector is as "intricate," or has as many "moving parts" or clients whose needs are as complicated. ${ }^{18}$ It is not surprising that many of the people at the vanguard of efforts to plan and deliver system-wide change have a background in systems design and engineering. ${ }^{16}$

What this shows is that to deliver improvement it is not enough to have a good grasp of how to apply quality improvement approaches and tools. Good relational skills matter just as much, if not more, ${ }^{19}$ as do the capacity to navigate the system and to build relationships and alliances with key stakeholders. 
Partnership working, therefore, is at the heart of all effective improvement interventions.

Competing interests: We have read the BMJ policy on competing interests and have the following interests to declare: none

Provenance and peer review: This article is part of a series commissioned by The BM/based on ideas generated by a joint editorial group with members from the Health Foundation and The BMJ, including a patient/carer. The BM/retained full editorial control over external peer review, editing, and publication. Open access fees and The BMJS quality improvement editor post are funded by the Health Foundation.

1 Smith I, Hicks C, McGovern T. Adapting Lean methods to facilitate stakeholder engagement and co-design in healthcare. BMJ2020;368:m35. doi: 10.1136/bmj.m35 pmid: 31992550

2 Jones B, Kwong E, Warburton W. Quality improvement made simple: what everyone should know about healthcare quality improvement. Health Foundation, 2021.

3 Health Foundation. Improvement projects that we fund. https://www.health.org.uk/what-wedo/supporting-health-care-improvement/improvement-projects-that-we-fund

4 Silver SA, McQuillan R, Harel Z, etal. How to sustain change and support continuous quality improvement. Clin J Am Soc Nephrol2016;11:916-24. doi: 10.2215/CJN.11501015 pmid: 27016498

5 Foy R, Skrypak M, Alderson S, etal. Revitalising audit and feedback to improve patient care. BMJ 2020;368:m213. doi: 10.1136/bmj.m213 pmid: 32107249

6 Dixon-Woods M. How to improve healthcare improvement-an essay by Mary Dixon-Woods. BMJ 2019;367:15514. doi: 10.1136/bmj.l5514 pmid: 31575526

7 Fulop NJ, Ramsay AIG. How organisations contribute to improving the quality of healthcare. BMJ 2019;365:11773. doi: 10.1136/bmj.l1773 pmid: 31048322

8 Drew JR, Pandit M. Why healthcare leadership should embrace quality improvement. BMJ 2020;368:m872. doi: 10.1136/bmj.m872 pmid: 32234838

9 Batalden P. Getting more health from healthcare: quality improvement must acknowledge patient coproduction-an essay by Paul Batalden. BMJ2018;362:k3617doi: 10.1136/bmj.k3617.

10 de longh A, Erdmann S. Better healthcare must mean better for patients and carers. BMJ 2018;361:k1877. doi: 10.1136/bmj.k1877 pmid: 29773551

11 Crisp H, Watt A, Jones B, Amevenu D, Warburton W. Improving flow along care pathways: learning from the Flow Coaching Academy Programme. Health Foundation, 2020doi: 10.37829/HF-2020-104.

12 Coughlan C, Manek N, Razak Y, Klaber RE. How to improve care across boundaries. BMJ 2020;369:m1045. doi: 10.1136/bmj.m1045 pmid: 32245848

13 Burgess N, Currie G, Crump B, Richmond J, Johnson M. Improving together: collaboration needs to start with regulators. BMJ2019;367:16392. doi: 10.1136/bmj.16392 pmid: 31776111

14 Delgado P, Binzer K, Shah A, Ekberg J, Arrieta J, Allwood D. Accelerating population health improvement. BMJ 2021;373:n966. doi: 10.1136/bmj.n966 pmid: 34103302

15 Mathews SC, Pronovost PJ. The need for systems integration in health care. JAMA2011;305:934-5. doi: 10.1001/jama.2011.237 pmid: 21364143

16 Macrae C, Stewart K. Can we import improvements from industry to healthcare?BMJ 2019;364:11039. doi: 10.1136/bmj.l1039 pmid: 30898765

17 Mannion R, Davies H. Understanding organisational culture for healthcare quality improvement. BMJ2018;363:k4907. doi: 10.1136/bmj.k4907 pmid: 30487286

18 Braithwaite J. Changing how we think about healthcare improvement. BMJ2018;361:k2014. doi: 10.1136/bmj.k2014 pmid: 29773537

19 Jones B, Vaux E, Olsson-Brown A. How to get started in quality improvement. BMJ 2019;364:k5408. doi: 10.1136/bmj.k5437 pmid: 30655245 